\title{
Trends in the Prevalence of Obesity and Its Phenotypes Based on the Korea National Health and Nutrition Examination Survey from 2007 to 2017 in Korea
}

\author{
Sang Ouk Chin ${ }^{1}$, You-Cheol Hwang ${ }^{2}$, Hong-Yup Ahn³ , Ji Eun Jun², In-Kyung Jeong², Kyu Jeung Ahn², Ho Yeon Chung² \\ ${ }^{1}$ Department of Endocrinology and Metabolism, Kyung Hee University Hospital, College of Medicine, Kyung Hee University, Seoul, \\ ${ }^{2}$ Department of Endocrinology and Metabolism, Department of Medicine, Kyung Hee University Hospital at Gangdong, College of Medicine, Kyung Hee \\ University, Seoul, \\ ${ }^{3}$ Department of Statistics, Dongguk University, Seoul, Korea
}

This study used data from the Korea National Health and Nutrition Examination Survey IV-VII from 2007 to identify the prevalence of obesity and its phenotypes (metabolically unhealthy obesity [MUO] and metabolically healthy obesity [MHO]) and their secular changes. The prevalence of obesity in Korea increased with significant secular changes observed $(\beta=0.326, P$ trend $<0.01)$ between 2007 and 2017, and especially in men $(\beta=0.682, P$ trend $<0.001)$ but not in women. The changes in the prevalence of obesity during the study period were different between men and women $(P=0.001)$. The prevalence of MUO significantly increased only in men $(\beta=0.565, P$ trend $<0.01)$, while that of MHO increased only in women $(\beta=0.179, P<0.05)$, especially in the younger age group $(\beta=0.308, P<0.01)$.

Keywords: Korea; Obesity; Prevalence

\section{INTRODUCTION}

The epidemiology of obesity in Korean adults is unique when compared to other countries. The prevalence of obesity is higher in men than women. In addition, while the prevalence of obesity has increased in men, but not in women during the past two decades [1]. However, no previous studies have investigated the long-term changes in epidemiologic data of obesity phenotypes, such as metabolically healthy obesity (MHO) and metabolically unhealthy obesity (MUO) in Korean adults or other populations. This study was designed to determine the secular changes in the prevalence of obesity and its phenotypes in Korean adults by using the data from the Korea National Health and Nutrition Examination Survey (KNHANES [2,3]).

\section{METHODS}

Obesity was defined as BMI $\geq 25.0 \mathrm{~kg} / \mathrm{m}^{2}$ [4], and the obesity phenotype was defined based on the criteria proposed by the National Cholesterol Education Program and Adult Treatment Panel III (NCEP: ATPIII) for the diagnosis of metabolic syndrome (MetS) [5]. According to these criteria, participants with obesity were divided into the $\mathrm{MHO}$ (BMI $\geq 25.0 \mathrm{~kg} / \mathrm{m}^{2}$ and 0 or 1 MetS traits) or MUO (BMI $\geq 25.0 \mathrm{~kg} / \mathrm{m}^{2}$ and $\geq 2 \mathrm{MetS}$ traits) groups [6]. In addition, if participants had cardiovascular diseases (CVDs) based on the survey in the KNHANES, they were regarded as MUO, regardless of the number of MetS traits. For those with BMI $<25.0 \mathrm{~kg} / \mathrm{m}^{2}$, they were classified as either metabolically healthy non-obesity (MHNO) or metabolically unhealthy non-obesity (MUNO) according to the
Corresponding author: You-Cheol Hwang (D) https://orcid.org/0000-0003-4033-7874 Department of Endocrinology and Metabolism, Department of Medicine, Kyung Hee University Hospital at Gangdong, College of Medicine, Kyung Hee University, 892

Dongnam-ro, Gangdong-gu, Seoul 05278, Korea

E-mail:khmcilyong@naver.com
This is an Open Access article distributed under the terms of the Creative Commons Attribution Non-Commercial License (https://creativecommons.org/licenses/by-nc/4.0/) which permits unrestricted non-commercial use, distribution, and reproduction in any medium, provided the original work is properly cited. 
number of traits for MetS based on NCEP: ATPIII criteria. For the sensitivity analysis, we used more stringent criteria to define the obesity phenotype. We excluded waist circumference from the definition, and $\mathrm{MHO}$ was defined as having none of the four remaining MetS traits [7,8]. Similarly, those with CVD were regarded as MUO for the sensitivity analysis. Definitions of these morbidities were described previously [2].

The age-standardized prevalence was estimated using the 2005 Korean Census population data to avoid bias due to changes in population structures across phases. The linear-bylinear association analysis was used to calculate the $P$ value for the trend. The regression coefficient $\beta$ was calculated to demonstrate the annual change in the prevalence of obesity and its phenotypes using weighted least square regression. To determine whether trends in the prevalence of obesity and its phenotypes differed by sex and age groups, the presence of interaction was assessed in multivariate models by testing the significance of first-order interaction terms. The analyses were performed using R statistical software version 2.14.2 (The R Foundation, Vienna, Austria; http://www.r-project.org). $P$ values $<0.05$ were considered statistically significant. This study was also approved by the Institutional Review Board of Kyung Hee University Hospital at Gangdong (KHNMC IRB 2020-04-025). When each KNHANES was conducted, all of the participants were informed that they had been randomly chosen to participate in the survey with the right to refuse to participate in the further analyses, and signed informed consents.

\section{RESULTS}

Approximately 35-42 million participants per year were included in this analysis (Supplementary Table 1). The total prevalence of obesity in Korea was $32.1 \%$ in 2007 , which increased to $34.4 \%$ in 2017 (Supplementary Table 2). This secular change was found to be statistically significant $(\beta=0.326, P$ trend $<0.01$ ) (Table 1, Fig. 1A, Supplementary Table 2). In the 20 to 39 years age group, the prevalence of obesity significantly increased between 2007 and $2017(\beta=0.602, P$ trend $<0.001)$ (Table 1). According to the sub-analysis by sex, the prevalence in men significantly increased from $36.7 \%$ in 2007 to $42.3 \%$ in 2017 ( $\beta=0.682, P$ trend $<0.001$ ) (Table 1, Fig. 1B, Supplementary Table 2$)$, and this change was significant across all age groups (Table 1). Conversely, significant changes were not observed in women during the same period $(\beta=-0.017, P$ trend $=0.880$ ) (Table 1, Fig. 1C, Supplementary Table 2). The subgroup analysis according to age group also revealed nonsignificant changes in women (Table 1). The change in the prevalence of obesity between 2007 and 2017 appeared to be significantly different between men and women $(P=0.001)$ (Table 1), even in the subgroup analyses by age group (Table 1 ).

The prevalence of MUO did not show a significant secular change during the study period ( $P$ trend $=0.150)$ (Supplementary Table 2), except in the 20 to 39 years age group $(\beta=0.382$, $P$ trend $<0.01$ ) (Table 1, Supplementary Table 2). The subgroup analysis according to sex demonstrated a significant increase in the prevalence of MUO in men $(\beta=0.565, P$ trend $<0.01$ ) (Table 1, Fig. 1B, Supplementary Table 2), which remained significant across all age groups, except in the 40 to 59 years age group (Table 1). In contrast, the prevalence of MUO showed a decreasing trend among women in all age groups, although it did not reach statistical significance (Table 1). Similar to the prevalence of obesity, the secular change in the prevalence of MUO between 2007 and 2017 was significantly different between men and women $(P=0.002)$ (Table 1$)$. This differ-

Table 1. Secular trends of obesity and its phenotypes at different age groups between 2007 and 2017 and its comparison between men and women

\begin{tabular}{|c|c|c|c|c|c|c|c|c|c|c|c|c|}
\hline & \multicolumn{4}{|c|}{ Obesity } & \multicolumn{4}{|c|}{ MUO } & \multicolumn{4}{|c|}{ MHO } \\
\hline & \multicolumn{3}{|c|}{$\beta^{\mathrm{a}}$} & \multirow{2}{*}{$P$ value ${ }^{\mathrm{b}}$} & \multicolumn{3}{|c|}{$\beta^{\mathrm{a}}$} & \multirow{2}{*}{$P$ value ${ }^{\mathrm{b}}$} & \multicolumn{3}{|c|}{$\beta^{\mathrm{a}}$} & \multirow{2}{*}{$P$ value } \\
\hline & Total & Men & Women & & Total & Men & Women & & Total & Men & Women & \\
\hline All age, yr & $0.326^{\mathrm{d}}$ & $0.682^{\mathrm{d}}$ & -0.017 & 0.001 & 0.193 & $0.565^{\mathrm{d}}$ & -0.180 & 0.002 & $0.160^{\mathrm{d}}$ & 0.129 & $0.179^{c}$ & 0.650 \\
\hline 20-39 & $0.602^{\mathrm{d}}$ & $0.874^{\mathrm{d}}$ & 0.306 & 0.019 & $0.382^{\mathrm{d}}$ & $0.739^{\mathrm{d}}$ & 0.008 & 0.004 & $0.219^{c}$ & 0.131 & $0.308^{\mathrm{d}}$ & 0.240 \\
\hline $40-59$ & 0.040 & $0.449^{c}$ & -0.359 & 0.008 & -0.018 & 0.302 & -0.374 & 0.034 & 0.108 & 0.121 & 0.057 & 0.670 \\
\hline$>60$ & 0.242 & $0.740^{c}$ & -0.035 & 0.023 & 0.199 & $0.723^{c}$ & -0.128 & 0.036 & 0.041 & 0.034 & 0.084 & 0.740 \\
\hline
\end{tabular}

MUO, metabolically unhealthy obesity; MHO, metabolically healthy obesity.

${ }^{a}$ Regression coefficient $\beta$ demonstrates the annual change of obesity and its phenotype prevalence by use of weighted least square regression,

${ }^{b}$ Difference of regression coefficient $\beta$ between men and women, ${ }^{c} P<0.05,{ }^{\mathrm{d}} P<0.01$ statistical significance for regression coefficient $\beta$. 

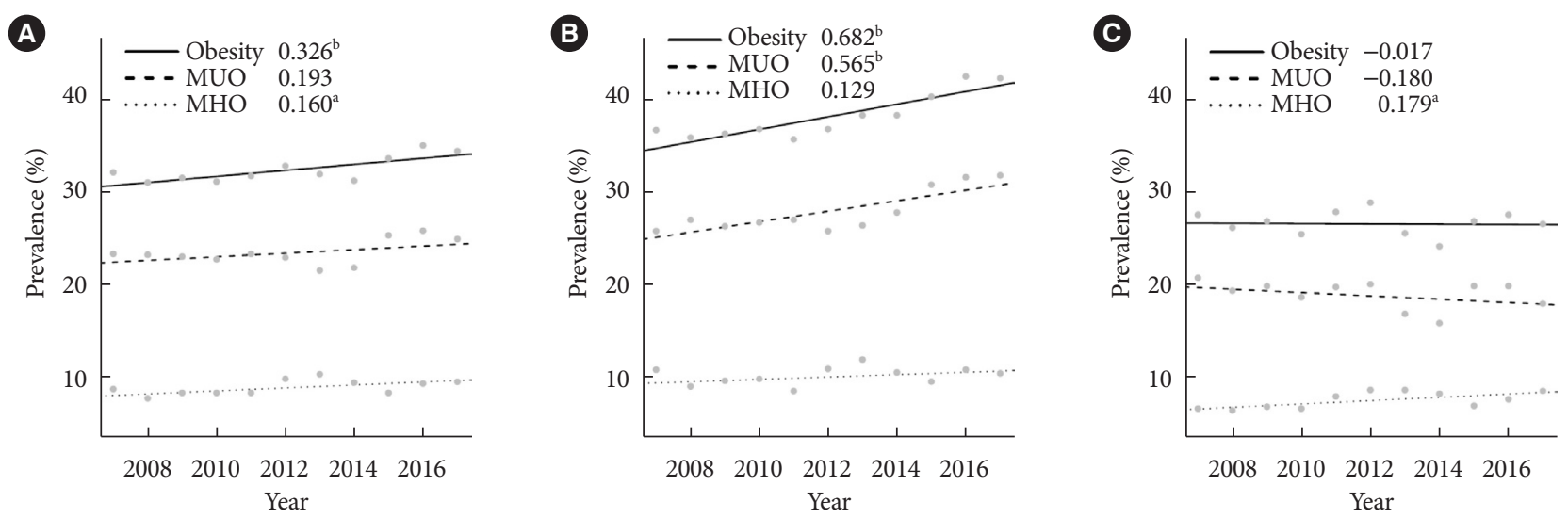

Fig. 1. Secular changes in the prevalence of obesity and its phenotypes in Korea between 2007 and 2017. The numbers shown in each figure represent the regression coefficient $\beta$ demonstrating the annual change of obesity and its phenotype prevalence by use of weighted least square regression. (A) All participants, (B) men, (C) women. MUO, metabolically unhealthy obesity; MHO, metabolically healthy obesity. ${ }^{\mathrm{a}} \mathrm{P}<0.05,{ }^{\mathrm{b}} \mathrm{P}<0.01$.

ence between sex remained its significance in the subgroup analyses according to each age group (Table 1). The sensitivity analysis similarly showed a significant increase in the prevalence of MUO in men across all age groups, but no significant change in the prevalence in women, and a significantly different secular change according to sex (Supplementary Table 3).

The prevalence of MHO significantly increased among all participants $(\beta=0.160, P<0.05)$ (Table 1, Fig. $1 \mathrm{~A}$, Supplementary Table 2), especially in the 20 to 39 years age group ( $\beta=$ $0.219, P<0.05$ ) (Table 1). Although the prevalence of MHO did not significantly change in men, that in women and especially those in the 20 to 39 years age group significantly increased ( $\beta=0.179, P<0.05$; Table 1, Fig. $1 \mathrm{C}$, Supplementary Table 2$)$ and $(\beta=0.308, P<0.01$; Table 1$)$. The changes in the prevalence of $\mathrm{MHO}$ were not significantly different between men and women during the study period $(P=0.650)$ (Table 1). The subgroup analyses by age group did not reveal any significant differences between sex in the prevalence of MHO (Table 1). The sensitivity analysis showed similar results: significant increase in the prevalence of MHO only in women across all age groups and in those aged 20 to 39 years. Its secular change between 2007 and 2017 was not significantly different according to sex (Supplementary Table 3).

As for the MHNO and MUNO, women in most age groups showed the increase in prevalence of MHNO and decrease in that of MUNO while men did not show significant change. The secular change only in the MHNO showed significant difference between sex at most of age groups (Supplementary Table 4).

\section{DISCUSSION}

Traditionally, the prevalence of obesity was known to be higher in women than in men, and the increase in its prevalence was usually higher in women than in men [9], and MHO was also reported to be more common in women than in men, and to be more likely to occur in younger people [10-13]. However, recently, sex difference in obesity is becoming closer in East Asian mega countries with high incomes such as Korea, China, and Japan [9], which is consistent with the findings of the present study. These changes may be viewed as promising in the epidemiology of obesity, especially among Korean women. To determine the possible causes of secular trends in the prevalence of obesity in Korea, we also analyzed data on various nutritional and lifestyle factors from the KNHANES data during the study period (data not shown), but unfortunately significant factors to explain this trend were not found. It should be addressed that previous studies have reported inconsistent results on the clinical importance of dietary habits or physical activities to predict the presence of MHO [14-16]. Very interestingly, the secular changes in percentage of traits satisfied to define the MetS were additionally analyzed according to sex and age group between 2007 and 2017 (Supplementary Table 5). The percentage of subjects with low high density lipoprotein cholesterol decreased and that with high fasting blood glucose increased. Only men notably demonstrated the additional increase in the percentage of subjects having hypertriglyceridemia. This may provide possible explanation for the 
increased prevalence of $\mathrm{MHO}$ only in men.

In conclusion, we found that the prevalence of obesity increased from 2007 to 2017, mostly in men, with a concomitant increase in the prevalence of MUO. Although the prevalence of obesity in women did not change, a significant increase in the prevalence of $\mathrm{MHO}$ was observed, especially in their younger age group.

\section{SUPPLEMENTARY MATERIALS}

Supplementary materials related to this article can be found online at https://doi.org/10.4093/dmj.2021.0226.

\section{CONFLICTS OF INTEREST}

You-Cheol Hwang was editorial board member of the Diabetes \& Metabolism Journal from 2020 to 2021. Hong-Yup Ahn has been statistical advisor of the Diabetes \& Metabolism Journal since 2021. In-Kyung Jeong was editor in chief of the Diabetes \& Metabolism Journal from 2020 to 2021. They were not involved in the review process of this article. Otherwise, there was no conflict of interest.

\section{AUTHOR CONTRIBUTIONS}

Conception or design: Y.C.H., J.E.J., I.K.J., K.J.A., H.Y.C. Acquisition, analysis, or interpretation of data: S.O.C., Y.C.H., H.Y.A.

Drafting the work or revising: S.O.C.

Final approval of the manuscript: S.O.C., Y.C.H., H.Y.A., J.E.J., I.K.J., K.J.A., H.Y.C.

\section{ORCID}

Sang Ouk Chin https://orcid.org/0000-0001-5914-3653

You-Cheol Hwang https://orcid.org/0000-0003-4033-7874

\section{FUNDING}

None

\section{ACKNOWLEDGMENTS}

None

\section{REFERENCES}

1. Jaacks LM, Vandevijvere S, Pan A, McGowan CJ, Wallace C, Imamura F, et al. The obesity transition: stages of the global epidemic. Lancet Diabetes Endocrinol 2019;7:231-40.

2. Chin SO, Rhee SY, Chon S, Hwang YC, Jeong IK, Oh S, et al. Sarcopenia is independently associated with cardiovascular disease in older Korean adults: the Korea National Health and Nutrition Examination Survey (KNHANES) from 2009. PLoS One 2013;8:e60119.

3. Lee YH, Kim SU, Song K, Park JY, Kim DY, Ahn SH, et al. Sarcopenia is associated with significant liver fibrosis independently of obesity and insulin resistance in nonalcoholic fatty liver disease: nationwide surveys (KNHANES 2008-2011). Hepatology 2016;63:776-86.

4. WHO Expert Consultation. Appropriate body-mass index for Asian populations and its implications for policy and intervention strategies. Lancet 2004;363:157-63.

5. Grundy SM, Brewer HB Jr, Cleeman JI, Smith SC Jr, Lenfant C; American Heart Association, et al. Definition of metabolic syndrome: report of the National Heart, Lung, and Blood Institute/American Heart Association conference on scientific issues related to definition. Circulation 2004;109:433-8.

6. Hwang YC, Cho IJ, Jeong IK, Ahn KJ, Chung HY. Differential association between sarcopenia and metabolic phenotype in Korean young and older adults with and without obesity. Obesity (Silver Spring) 2017;25:244-51.

7. Guo F, Garvey WT. Cardiometabolic disease risk in metabolically healthy and unhealthy obesity: stability of metabolic health status in adults. Obesity (Silver Spring) 2016;24:516-25.

8. Al-Khalidi B, Kimball SM, Kuk JL, Ardern CI. Metabolically healthy obesity, vitamin $\mathrm{D}$, and all-cause and cardiometabolic mortality risk in NHANES III. Clin Nutr 2019;38:820-8.

9. Chooi YC, Ding C, Magkos F. The epidemiology of obesity. Metabolism 2019;92:6-10.

10. Lin H, Zhang L, Zheng R, Zheng Y. The prevalence, metabolic risk and effects of lifestyle intervention for metabolically healthy obesity: a systematic review and meta-analysis. A PRISMA-compliant article. Medicine (Baltimore) 2017;96: e8838.

11. Slagter SN, Corpeleijn E, van der Klauw MM, Sijtsma A, SwartBusscher LG, Perenboom C, et al. Dietary patterns and physical activity in the metabolically (un)healthy obese: the Dutch Lifelines cohort study. Nutr J 2018;17:18.

12. Velho S, Paccaud F, Waeber G, Vollenweider P, Marques-Vidal 
P. Metabolically healthy obesity: different prevalences using different criteria. Eur J Clin Nutr 2010;64:1043-51.

13. Hinnouho GM, Czernichow S, Dugravot A, Nabi H, Brunner EJ, Kivimaki M, et al. Metabolically healthy obesity and the risk of cardiovascular disease and type 2 diabetes: the Whitehall II cohort study. Eur Heart J 2015;36:551-9.

14. Phillips CM. Metabolically healthy obesity: definitions, determinants and clinical implications. Rev Endocr Metab Disord
2013;14:219-27.

15. Prince RL, Kuk JL, Ambler KA, Dhaliwal J, Ball GD. Predictors of metabolically healthy obesity in children. Diabetes Care 2014;37:1462-8.

16. Heinzle S, Ball GD, Kuk JL. Variations in the prevalence and predictors of prevalent metabolically healthy obesity in adolescents. Pediatr Obes 2016;11:425-33. 